\title{
Using the nonlinear control of anaesthesia-induced hypersensitivity of EEG at burst suppression level to test the effects of radiofrequency radiation on brain function
} Tarmo Lipping*1, Michael Rorarius², Ville Jäntti³, Kari Annala², Ari Mennander ${ }^{2}$, Rain Ferenets ${ }^{1}$, Tommi Toivonen ${ }^{4}$, Tim Toivo ${ }^{4}$, Alpo Värri ${ }^{1}$ and Leena Korpinen*1,5

\author{
Address: ${ }^{1}$ Tampere University of Technology, Pori, Finland, ${ }^{2}$ Tampere University Hospital, Tampere, Finland, ${ }^{3}$ Seinäjoki Central Hospital, \\ Seinäjoki, Finland, ${ }^{4}$ STUK, Radiation and Nuclear Safety Authority, Helsinki, Finland and ${ }^{5}$ Faculty of Medicine, University of Tampere, Tampere, \\ Finland \\ Email: Tarmo Lipping* - tarmo.lipping@tut.fi; Michael Rorarius - Michael.Rorarius@pshp.fi; Ville Jäntti - Ville.Jantti@uta.fi; \\ Kari Annala - kari.annala@pshp.fi; Ari Mennander - Ari.Mennander@pshp.fi; Rain Ferenets - rain.ferenets@gmail.com; \\ Tommi Toivonen - Tommi.Toivonen@stuk.fi; Tim Toivo - Tim.Toivo@stuk.fi; Alpo Värri - Alpo.Varri@tut.fi; \\ Leena Korpinen* - leena.korpinen@tut.fi \\ * Corresponding authors
}

This article is available from: http://www.nonlinearbiomedphys.com/content/3/l/5

(c) 2009 Lipping et al; licensee BioMed Central Ltd.

This is an Open Access article distributed under the terms of the Creative Commons Attribution License (http://creativecommons.org/licenses/by/2.0), which permits unrestricted use, distribution, and reproduction in any medium, provided the original work is properly cited.

\begin{abstract}
Background: In this study, investigating the effects of mobile phone radiation on test animals, eleven pigs were anaesthetised to the level where burst-suppression pattern appears in the electroencephalogram (EEG). At this level of anaesthesia both human subjects and animals show high sensitivity to external stimuli which produce EEG bursts during suppression. The burstsuppression phenomenon represents a nonlinear control system, where low-amplitude EEG abruptly switches to very high amplitude bursts. This switching can be triggered by very minor stimuli and the phenomenon has been described as hypersensitivity. To test if also radio frequency (RF) stimulation can trigger this nonlinear control, the animals were exposed to pulse modulated signal of a GSM mobile phone at $890 \mathrm{MHz}$. In the first phase of the experiment electromagnetic field (EMF) stimulation was randomly switched on and off and the relation between EEG bursts and EMF stimulation onsets and endpoints were studied. In the second phase a continuous RF stimulation at $31 \mathrm{~W} / \mathrm{kg}$ was applied for 10 minutes. The ECG, the EEG, and the subcutaneous temperature were recorded.
\end{abstract}

Results: No correlation between the exposure and the EEG burst occurrences was observed in phase I measurements. No significant changes were observed in the EEG activity of the pigs during phase II measurements although several EEG signal analysis methods were applied. The temperature measured subcutaneously from the pigs' head increased by $1.6^{\circ} \mathrm{C}$ and the heart rate by $14.2 \mathrm{bpm}$ on the average during the $10 \mathrm{~min}$ exposure periods.

Conclusion: The hypothesis that RF radiation would produce sensory stimulation of somatosensory, auditory or visual system or directly affect the brain so as to produce EEG bursts during suppression was not confirmed. 


\section{Background}

Indications of the RF-EMF originated changes in biological systems have been researched by exposing human volunteers, animals and cells to radio frequency (RF) fields as well as by performing epidemiological studies [1-6]. The studies on the effect of exposure to RF fields on brain functions and among others on behaviour of test animals have yielded contradictory results [7-13]. In many studies the applied RF frequencies and their modulation have not corresponded to the frequencies used by commercial mobile phones. The background radiation has complicated the evaluation of the results.

Animal studies are especially useful for experiments in which the exposure level exceeds the limits for human exposure (for exposure levels, see [14]). The use of high exposure levels is an essential element of toxicological testing (e.g., safety testing of chemicals): sufficiently high exposure levels are used to find a biological response, and lower exposures are then systematically tested to find the "safe" level (taking into account possible sensitivity differences between the test animals and humans). This kind of toxicological approach has not yet been applied for systematic testing of the effects of RF fields on brain function in animals.

Animal studies are also well suited for studying the hypothesized specific effects of amplitude-modulated RF fields on the central nervous system. If specific bioeffects of amplitude-modulated fields exist (for review, see [1]), they would be especially relevant for current digital mobile phone systems such as GSM. A few studies have reported effects of modulated RF fields on animal electroencephalogram (EEG) [15-17], but systematic research on this question is lacking, and the positive findings have not been followed up. In this study anaesthetised pigs are used as test animals. The pig, with its relatively large brain size, has proven to be one of the best options as a test animal when considering effects on the brain.

During anaesthesia the EEG shows less variability compared to the waking condition when sensory-motor activity, cognitive functions and mental activity among other factors might cause the properties of the signal to fluctuate in an unpredictable manner. Anaesthesia itself modulates the properties of the EEG, however, these changes are relatively well known. We have previously shown that during burst suppression, i.e., the phase of deep anaesthesia when periods of relatively flat EEG alternate with high amplitude mixed frequency bursts, the bursts can be provoked by somatosensory, photic and auditory stimuli $[18,19]$. In fact, minor stimuli such as vibration [20] or light touch [21-23] readily produce bursts. Bursts can be produced by both the onset and end of a train of stimuli with the latency remaining fairly constant. Burst suppression is closely related to slow wave sleep oscillations, which develop a nonlinear control system for intermittent suppressions. Suppression segments are essentially prolonged up-states of the cortical sleep oscillation [23]. In anaesthesia with volatile agents as well as ischemic brain damage this nonlinear, on-off, control system sometimes becomes reactive to minimal perturbations. This phenomenon has been called hypersensitivity of the anaesthesia-induced comatose brain [24,25]. We therefore hypothesized that this extremely sensitive phenomenon, representing a very sensitive nonlinear control system, might be an indicator of possible RF effects.

The objective of this study was to determine the effects of radiofrequency radiation from the GSM mobile phone system on the electrical activity of a pig brain at radiation levels above those accepted for human testing. The aims were to find the threshold for observable changes in the brain's electrical activity, to document the consequences of exposure above the threshold, and to study whether there is any difference between the effects of short exposure pulses and continuous exposure. The experiments were designed with two objectives in mind: 1) to study the reactivity of the EEG burst suppression pattern to the EMF stimulation and 2) to study the changes in the EEG signal due to continuous high power EMF radiation.

\section{Materials and methods}

Altogether 11 experiments were performed during the study. The experiments were carried out in two series with the first and the second experiment series including 5 and 6 animals, respectively. The animals were anaesthetized with isoflurane to the level where the burst suppression pattern occurs. The level of anaesthesia was controlled by visual inspection of the EEG signal in the course of the recordings. The first experiment series was recorded using Cadwell Easy Writer ${ }^{\mathrm{Tm}}$ of Cadwell Laboratories, Kennewick, WA, USA (sampling frequency $200 \mathrm{~Hz}$ ) and the second one with Biopac data acquisition system from Biopac Systems, Goleta, CA, USA (sampling frequency $1000 \mathrm{~Hz}$ ). The exposures were conducted using a setup described in [26]. The exposure source was a half wave dipole fed by an amplified signal of a computer-controlled mobile phone. The mobile phone was transmitting a typical pulse modulated GSM voice call signal at $890.2 \mathrm{MHz}$ (Figures 1 and 2). Each measurement consisted of two phases. During phase I the EMF stimulation was switched on and off in a random fashion while anaesthesia was kept at a level where the EEG suppression segments lasted 5 to 10 sec on the average. In phase II 10 min continuous stimulation was applied with the anaesthesia kept at a deep level with still continuous EEG. During the whole experiment the EEG, the electrocardiogram (ECG) and the stimulation marker were recorded. The EEG electrodes were implanted subcutaneously to obtain high quality signals. The functioning of the EEG and the ECG sensors in strong electromagnetic field was tested prior to the exposures by 


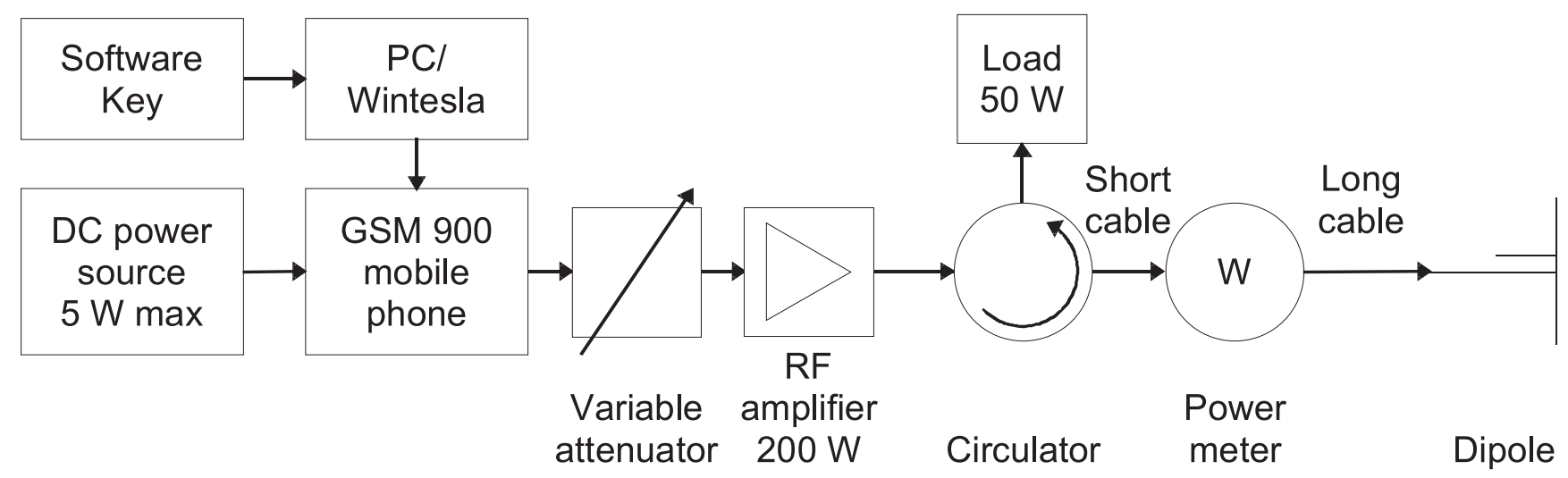

Figure I

Schematic diagram of the exposure system.

attaching the electrodes to a spherical dielectric phantom and irradiating it at maximum available power level (200 $\mathrm{W}$ peak). No interference was observed.

All animals received humane care in accordance with the Principles of Laboratory Animal Care formulated by the National Society for Medical Research and the Guide for the Care and Use of Laboratory Animals prepared by the Institute of Laboratory Animal Resources, National Research Council, and published by the National Academy Press, revised in 1996. The ethical evaluations of experiments were reviewed and approved by the Ethical Committee for Research Animal Care and Use of the University of Tampere.

\section{Phase I: reactivity of the burst-suppression pattern to EMF exposure}

During the about $10-20$ min period of phase I of the experiment the EMF stimulation source was switched on

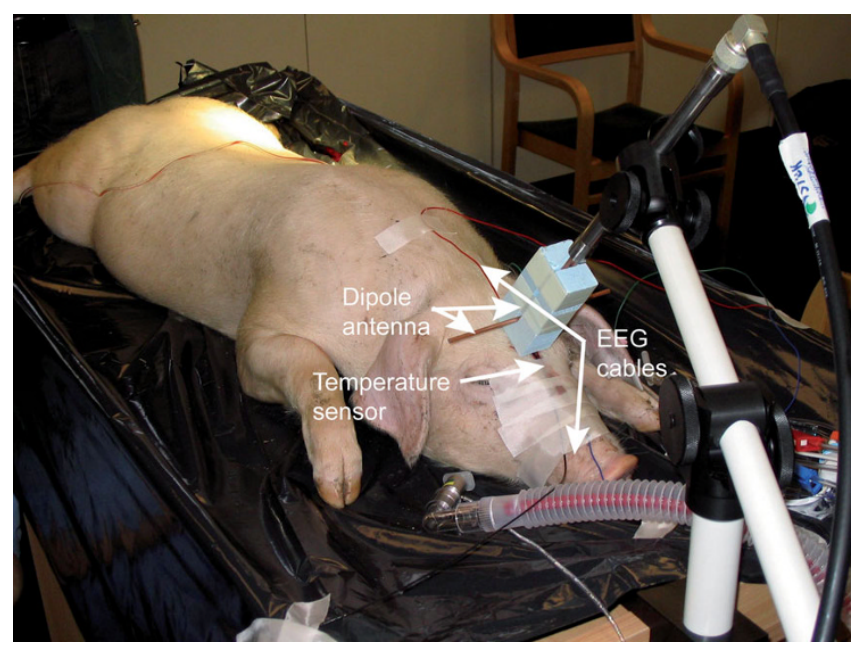

Figure 2

Recording setup. and off for 1-10 s in irregular intervals, first at the exposure level of $7.3 \mathrm{~W} / \mathrm{kg}$ and then at $31 \mathrm{~W} / \mathrm{kg}$ (maximum 10 $\mathrm{g}$ average [14]). A sample of the recorded signals is shown in Figure 3.

The EEG signal was analyzed visually to detect the onsets of bursts and suppression segments. Interburst intervals (IBI) were calculated and accepted for further analysis if their length remained below 100 seconds. The start- and end times of stimulation were identified from the marker curve. The stimulation start- and end times occurring during EEG bursts were discarded. Also, if after a stimulation onset the stimulation was switched off before an EEG burst started, the onset of this particular stimulation period was discarded. Correspondingly, if after a stimulation endpoint a new stimulation period was started before an EEG burst occurred the particular stimulation endpoint was discarded (e.g., the endpoint of the second stimulation period in Figure 3). For the remaining stimulation start- and endpoints the Stimulation Start to Burst Intervals (SSBIs) and Stimulation End to Burst Intervals (SEBIs) were calculated. The total number of detected IBIs and stimulation periods together with the number of IBIs, SSBIs and SEBIs considered valid for further analysis are given in Table 1.

\section{Phase II: 10 minute continuous exposure}

Following phase I, after a short period of no exposure, phase II of the experiment was carried out by switching on the EMF source for 10 minutes at the SAR level of $31 \mathrm{~W} /$ $\mathrm{kg}$. Anaesthesia was kept at lighter level at this phase so that the EEG was continuous with no burst-suppression pattern observed. The temperature was measured subcutaneously using a fluoro-optic sensor (Luxtron 750) and the temperature values were written down manually every minute during (and for several minutes after) the continuous exposure period. About 10-20 minutes after the EMF source was switched off the animal was sacrificed. 


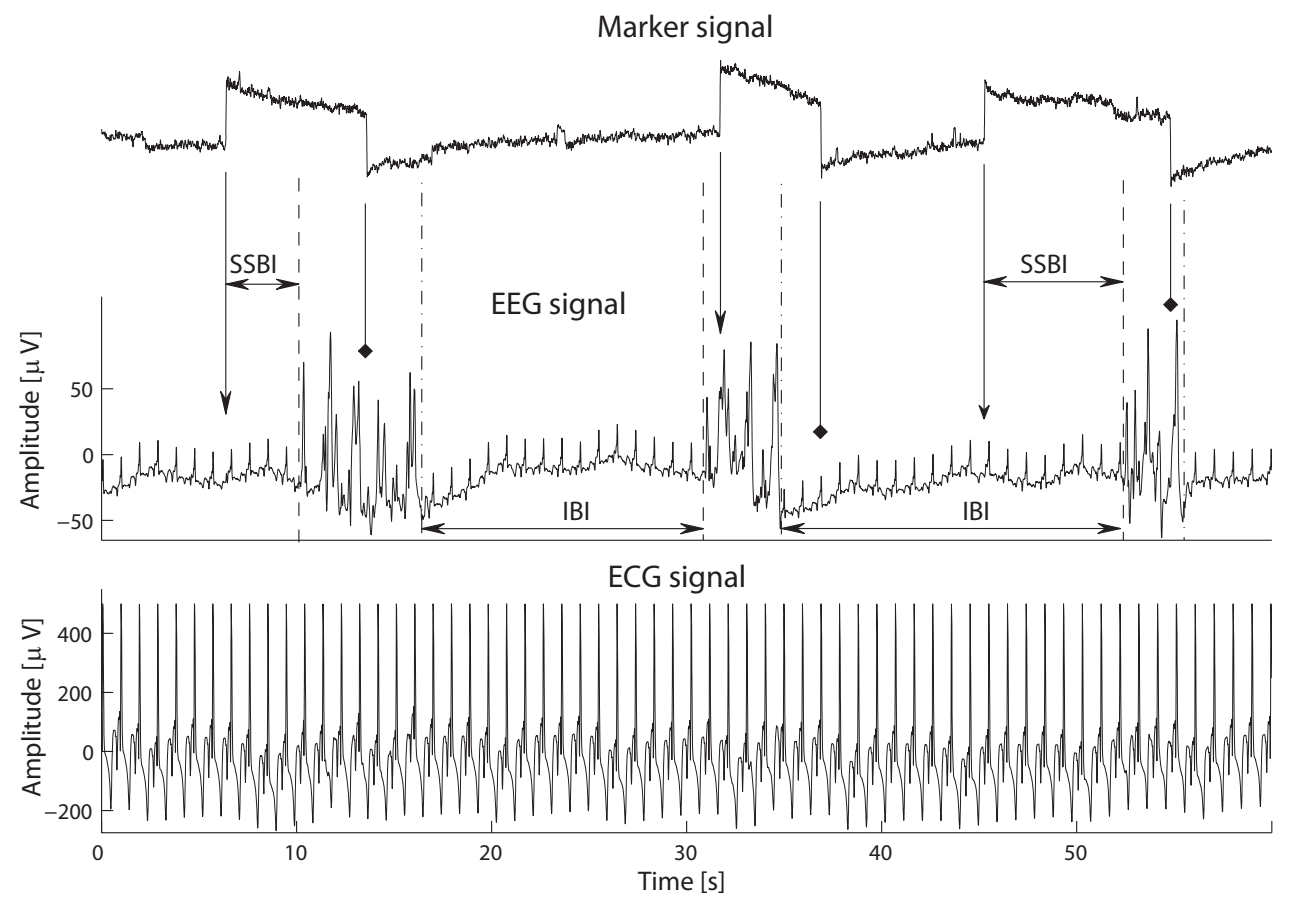

Figure 3

A sample of the recorded signals of phase I recording. The vertical arrows with $V$-shaped and diamond-shaped heads indicate the time instances of on- and off-switching the EMF source, respectively. Start- and end times of bursts are marked by dashed and dash-dotted lines, respectively. Three events, the endpoints of the first and the third stimulation period as well as the onset of the second stimulation period are discarded as they occur during bursts. Also, the endpoint of the second stimulation period is discarded because the EMF source is switched on again before a burst occurs. Two stimulation-start-to-burst intervals (SSBI) and two interburst intervals (IBI) are indicated in the figure. The EEG signal is contaminated by ECG artifact, however, the bursts can easily be detected by visual analysis. The ECG artifact was not present during the periods of continuous 10 minute exposure of phase II.

Table I: Statistics on interburst intervals (IBI), stimulation periods, stimulation start to burst intervals (SSBI) and stimulation end to burst intervals (SEBI).

\begin{tabular}{|c|c|c|c|c|c|}
\hline Recording nr. & Total nr of IBIs & $\begin{array}{c}\text { Nr of IBIs shorter than } 100 \\
\text { sec. }\end{array}$ & Nr of stimulation periods & Nr of valid SSBIs & Nr of valid SEBIs \\
\hline Set I Rec. I & 214 & 213 & 79 & 47 & 40 \\
\hline Set I Rec. 2 & 128 & 127 & 89 & 48 & 35 \\
\hline Set I Rec. 3 & 195 & 194 & 83 & 47 & 36 \\
\hline Set I Rec. 4 & 218 & 218 & 67 & 47 & 28 \\
\hline Set 2 Rec. 1 & 93 & 93 & 37 & 10 & 8 \\
\hline Set 2 Rec. 2 & 156 & 156 & 88 & 41 & 57 \\
\hline Set 2 Rec. 3 & 54 & 54 & 45 & 19 & 15 \\
\hline Set 2 Rec. 4 & 188 & 188 & 44 & 30 & 17 \\
\hline Set 2 Rec. 5 & 73 & 73 & 28 & 2 & 12 \\
\hline Set 2 Rec. 6 & 105 & 105 & 55 & 31 & 17 \\
\hline Total & 1424 & $|42|$ & 615 & 322 & 265 \\
\hline
\end{tabular}


Heart rate was obtained from the ECG signal off-line using a simple prefilter and compare-to-threshold algorithm. Due to some technical problems in the recordings, the analysis of phase II recordings could only be performed for the recordings of set 2 .

Six segments of half a minute duration were extracted from the EEG signal at the following phases of the experiment: 1) just before stimulation onset, 2) just after stimulation onset, 3) in the middle of the stimulation period, 4) just before the end of the stimulation period, 5) just after the end of the stimulation period, and 6) five minutes post-stimulus. The signal segments were low-pass filtered and decimated 10 times, the new sampling frequency being $100 \mathrm{~Hz}$. Relative power in the following EEG frequency bands was calculated: delta $(0.5-4 \mathrm{~Hz})$, theta (4$8 \mathrm{~Hz})$, alpha $(8-12 \mathrm{~Hz})$, beta $(12-20 \mathrm{~Hz})$, and gamma (20-45 Hz). The power spectrum was calculated using autoregressive model parameters (Yule-Walker algorithm). In addition, spectral entropy [27], approximate entropy [28], Higuchi fractal dimension [29], and Lempel-Ziv complexity [30] were estimated from the EEG signal segments.

\section{Results}

EMF exposure effects on the EEG

By visual inspection of the EEG recordings of phase I, no obvious correlation between the timing of the burst-suppression pattern and the switching of the EMF source was observed. For better insight the distributions of IBIs, SSBIs, and SEBIs were plotted (see Figure 4). While the distributions of SSBIs and SEBIs look similar, very few IBIs shorter than $0.5 \mathrm{~s}$ occur. Also, the distribution of IBIs is wider, which comes from the fact that stimulation startand end times coinciding with bursts were omitted. Mean IBIs, SSBIs and SEBIs were 4.76, 2.25, and 2.70 s, respectively. The shapes of the distributions of Figure 4 were further compared by obtaining quantile-quantile plots (see Figure 5). SSBIs and SEBIs follow very similar distributions (the right panel of the figure) if not taking into account a couple of outliers. The distribution of IBIs is more long-tailed compared to those of SSBIs and SEBIs, causing the data in the left and middle panels of Figure 5 to deviate from the straight line.

The results of the EEG analysis of phase II recordings are shown in Figure 6 and the statistical analysis is presented in Table 2. The results indicate that no significant changes can be observed in the calculated EEG measures due to the continuous EMF exposure. The recordings show quite different behaviour, however. In recordings 1 and 3 a clear peak in the power spectrum at around $7-9 \mathrm{~Hz}$ was observed. Also, the entropy measures were higher for these test animals. In some recordings certain measures like theta ratio (for recording 1) or approximate entropy (for recording 6) show clear correlation with the EMF exposure. However, these results do not repeat in other test animals.

\section{EMF exposure effects on heart rate and subcutaneous temperature}

Heart rate and temperature changes during the exposure periods for recordings of data set 2 are shown in Figure 7. The curves are aligned according to the onset of the expo-
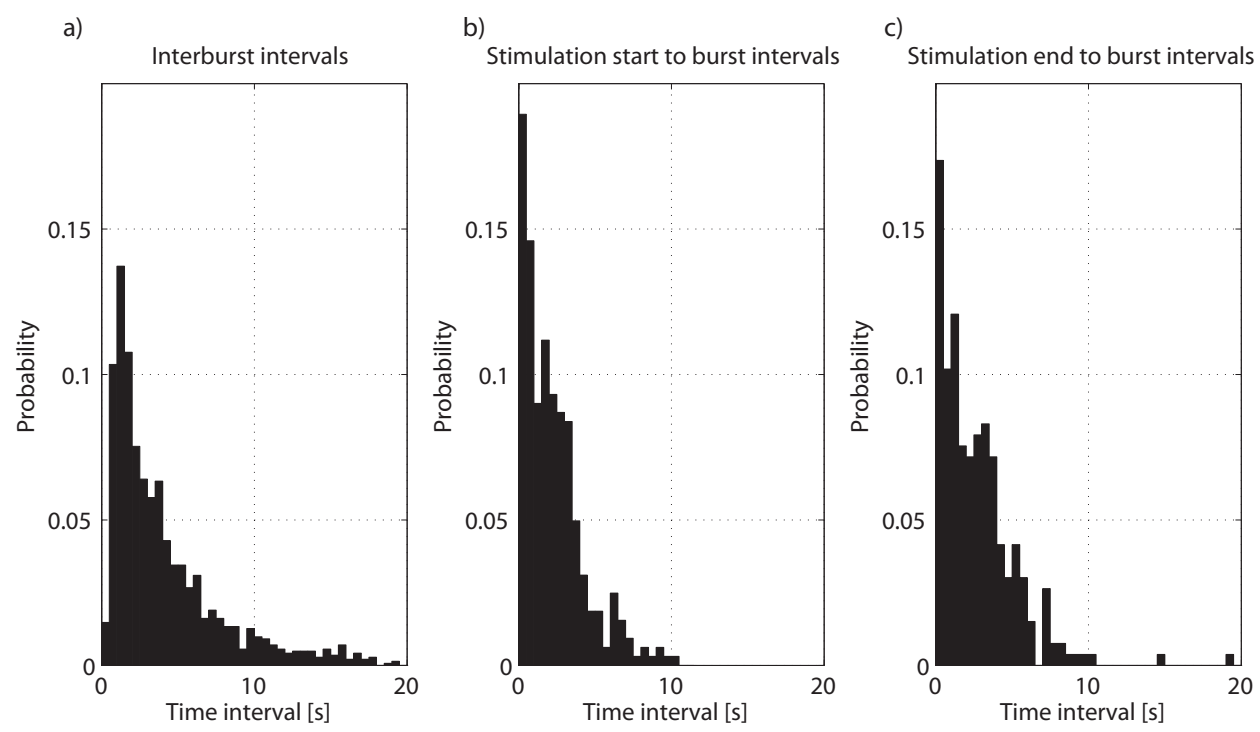

\section{Figure 4}

Distributions of: a) interburst intervals, b) stimulation start to burst intervals and c) stimulation end to burst intervals. $2.7 \%$ of the interburst intervals were longer than 20 seconds. 
a)

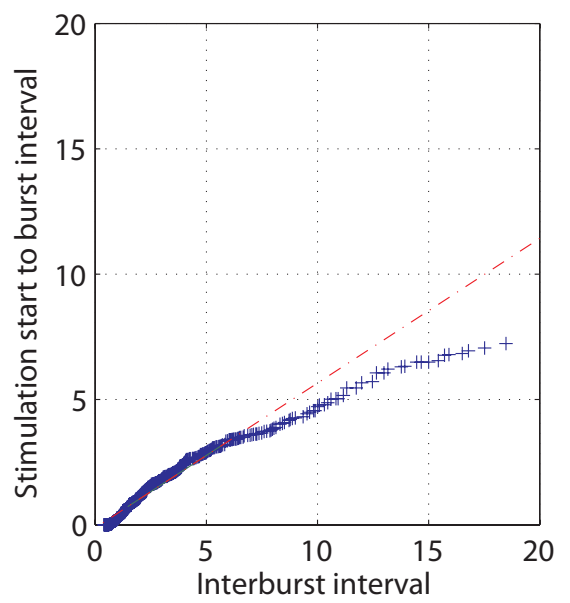

b)

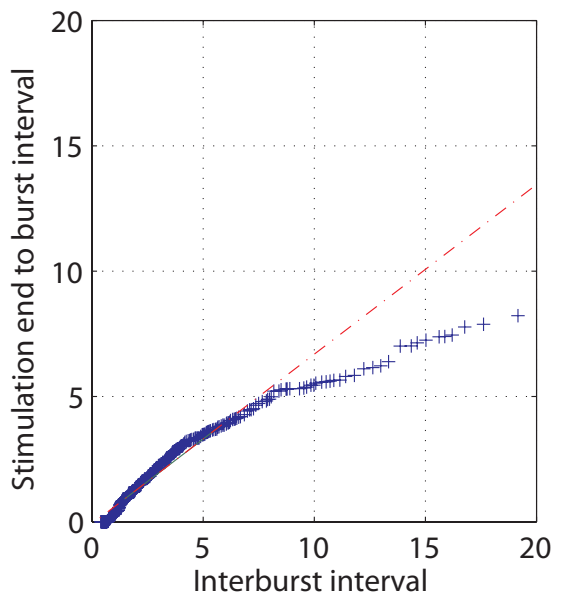

c)

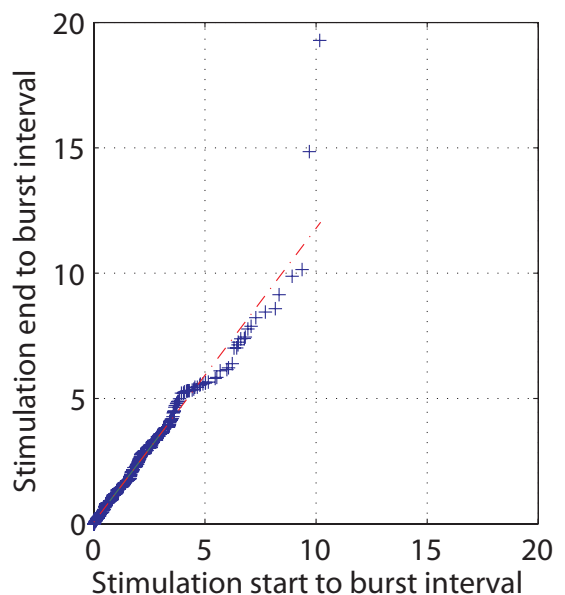

\section{Figure 5}

Quantile-quantile plots of the distributions shown in Figure 4: a) stimulation start to burst interval vs. interburst interval, b) stimulation end to burst interval vs. interburst interval, c) stimulation start to burst interval vs. stimulation end to burst interval.

sure period. The time instances at which exposure is switched on or off were obtained from the marker signal and are thus accurately aligned with the heart rate curve. There may be slight misalignment (up to about half a minute) between the temperature curves and the heart rate, however, as the temperature values were marked manually. During the 10 minute stimulation the subcutaneous temperature increased by 1.63 (standard deviation 0.89 , range $0.9 . .2 .9$ ) degrees. When the heart rate curves were fitted with straight lines over the stimulation period and the difference between the last and first values of the line were taken as the heart rate change during the stimulation, the average heart rate increase was calculated to be 14.15 (standard deviation 17.03, range $0.08 \ldots 45.57$ ) beats per minute. Although the heart rate and temperature increased during the exposure in all the recordings, no clear correlation between the magnitudes of these changes could be noted.

\section{Discussion}

Burst suppression pattern in the EEG signal is generated in the cerebral cortex. It has been shown that the cortex, when isolated from deep structures, produces burst suppression. This is also reflected by the fact that in brain damage and focal status epilepticus, focal burst suppression is sometimes seen. Even burst suppression recorded from depth electrodes is likely to be of cortical origin [23]. The bursts induced by minor somatosensory, auditory or photic stimuli are likely triggered by brainstem, as are the bursts induced in ischemic brain damage. The triggering sensory information may, thus, originate from auditory, somatosensory or visual systems and our experiment tests, whether radiation can produce similar triggering sensory effect.

The hypothesis that switching the EMF stimulation on or off would induce bursts during deep burst suppression level anaesthesia was not confirmed by our experiments. The shape of the distribution of interburst intervals was similar to that of the distributions of time intervals from the start and endpoints of the stimulus to the burst onset. This similarity of the distributions was further confirmed by quantile-quantile plots. The main difference in the shapes of the distributions was the considerably higher probability of very short intervals from stimulus start/end to burst onset compared to IBIs. This can be explained by the physiological refractory period after bursts, making IBIs of less than $1 \mathrm{~s}$ improbable in the spontaneous burst suppression pattern. Also, during visual annotation of burst start- and end times short suppression segments (< $1 \mathrm{~s})$ between bursts are often ignored. Omitting stimulation events during bursts might cause bias, but in our view this was the most reasonable way of handling these events. If there is already a burst going on while EMF source is switched on (or off), there is no way to tell if this particular switching had an influence on the bursting dynamics in the EEG.

The data recorded in this project does not give a comprehensive answer about the possible interaction between the EMF stimulation and the EEG bursting phenomenon as no sham recordings were available and the two phenomena, spontaneous bursting and possible interaction, could not be separated from one another. The similarity 
Delta Ratio

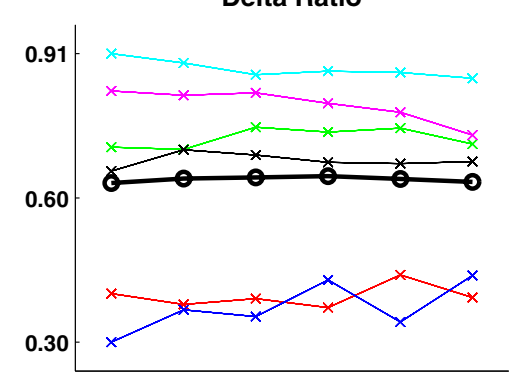

Beta Ratio

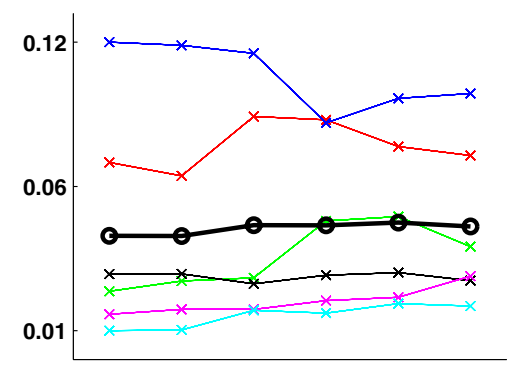

Spectral Entropy

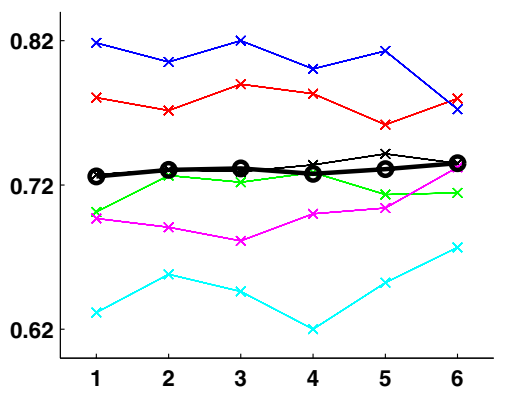

Theta Ratio

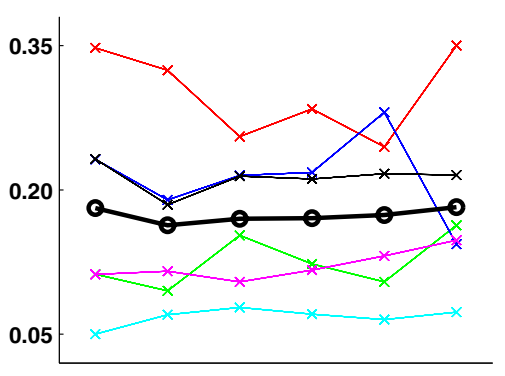

Gamma Ratio

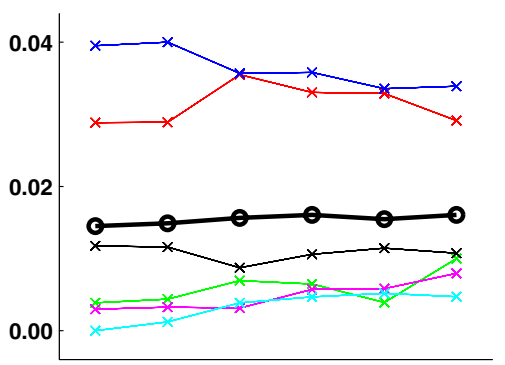

Lempel-Ziv Complexity

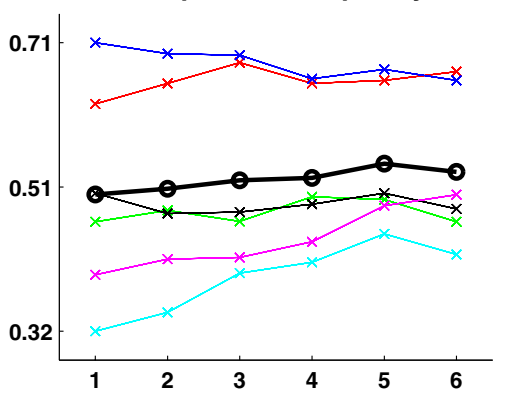

Alpha Ratio

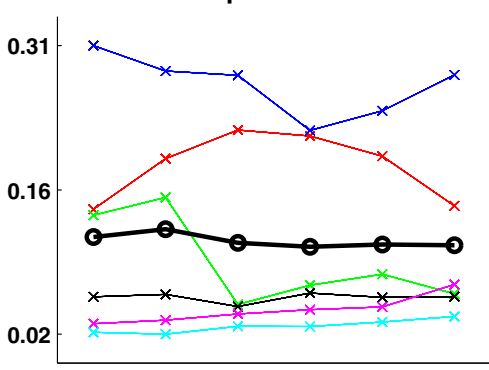

Higuchi Fractal Dimension

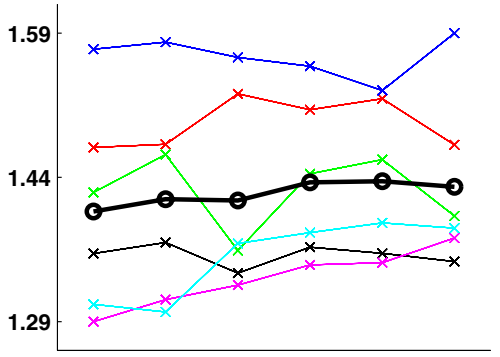

Approximate Entropy

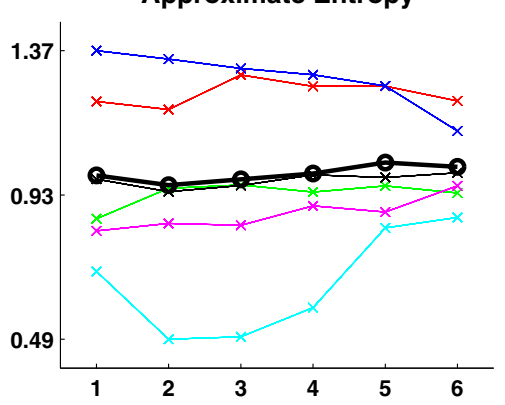

\section{Figure 6}

The results of the EEG analysis of phase II recordings. The curves correspond to the experiment animal recordings (recording I: red, recording 2: green, recording 3: blue, recording 4: black, recording 5: magenta, recording 6: cyan). The black line with circle markers indicates the mean over the recordings. All the measures are dimensionless, indicating either power ratio or signal complexity in arbitrary units. The subsegments are numbered according to the time flow of the experiments from pre-stimulus (segment I) to 5 min post-stimulus (segment 6).

of the distributions indicates, however, that if such kind of interaction exists, it is not obvious.

The frequency content of the EEG signal showed no significant changes related to the continuous EMF stimulation in phase II recordings. Various measures of signal entropy and complexity have recently been found to correlate with changes in the EEG due to, for example, deepening anaesthesia [31]. However, none of the measures applied in this study: Higuchi fractal dimension, spectral entropy, Lempel-Ziv complexity, or approximate entropy, revealed any trends during the stimulation. This might be surprising as a clear increase in temperature by about 1-2 degrees was observed. A possible reason for these results might be that the temperature was measured from below the scalp while it is possible that increased blood perfusion has compensated for the potential temperature increase due to the EMF power in deeper brain structures. This explanation is supported by the increase in heart rate. Unfortunately, blood pressure was not recorded in the experiments. Also, other variables like the anaesthetic effect, for example, were not fully controlled.

\section{Conclusion}

The exposure to $890 \mathrm{MHz}$ EMF stimulation at $31 \mathrm{~W} / \mathrm{kg}$ increased the subcutaneous temperature of the experi- 
Table 2: P-value obtained pairwise for all the calculated EEG measures and all the extracted segments using Wilcoxon ranksum test.

\begin{tabular}{|c|c|c|c|c|c|c|c|c|c|c|c|c|c|c|c|}
\hline & \multicolumn{5}{|c|}{ Delta } & \multicolumn{5}{|c|}{ Theta } & \multicolumn{5}{|c|}{ Alpha } \\
\hline & seg2 & seg3 & seg 4 & $\operatorname{seg} 5$ & seg6 & seg2 & seg3 & seg 4 & $\operatorname{seg} 5$ & seg6 & seg2 & seg3 & seg 4 & seg 5 & seg6 \\
\hline segI & 0.94 & 1.00 & 0.94 & 0.94 & 0.94 & 0.82 & 0.82 & 1.00 & 1.00 & 0.94 & 0.82 & 0.94 & 0.94 & 0.94 & 0.70 \\
\hline $\operatorname{seg} 2$ & & 1.00 & 1.00 & 0.94 & 0.94 & & 0.70 & 0.59 & 0.82 & 0.70 & & 0.94 & 0.94 & 1.00 & 1.00 \\
\hline seg3 & & & 1.00 & 0.94 & 0.94 & & & 1.00 & 0.94 & 1.00 & & & 1.00 & 0.82 & 0.48 \\
\hline seg4 & & & & 0.94 & 0.94 & & & & 1.00 & 0.82 & & & & 0.94 & 0.94 \\
\hline \multirow[t]{3}{*}{ seg5 } & & & & & 0.82 & & & & & 0.94 & & & & & 0.82 \\
\hline & \multicolumn{5}{|c|}{ Beta } & \multicolumn{5}{|c|}{ Gamma } & \multicolumn{5}{|c|}{ HFD } \\
\hline & seg2 & seg3 & seg4 & $\operatorname{seg} 5$ & seg6 & seg2 & seg3 & seg 4 & $\operatorname{seg} 5$ & seg6 & seg2 & seg3 & seg 4 & $\operatorname{seg} 5$ & seg6 \\
\hline segl & 0.94 & 0.82 & 0.70 & 0.59 & 0.59 & 0.82 & 0.70 & 0.59 & 0.59 & 0.59 & 0.70 & 0.82 & 0.59 & 0.59 & 0.59 \\
\hline seg2 & & 1.00 & 0.82 & 0.59 & 0.59 & & 0.94 & 0.59 & 0.70 & 0.59 & & 1.00 & 0.82 & 0.82 & 0.70 \\
\hline $\operatorname{seg} 3$ & & & 1.00 & 0.70 & 0.59 & & & 0.82 & 1.00 & 0.70 & & & 0.59 & 0.70 & 0.39 \\
\hline $\operatorname{seg} 4$ & & & & 0.82 & 1.00 & & & & 0.82 & 0.82 & & & & 0.94 & 0.94 \\
\hline \multirow[t]{3}{*}{$\operatorname{seg} 5$} & & & & & 0.94 & & & & & 0.82 & & & & & 1.00 \\
\hline & \multicolumn{5}{|c|}{ SPEN } & \multicolumn{5}{|c|}{ LZC } & \multicolumn{5}{|c|}{ APEN } \\
\hline & seg2 & seg3 & seg4 & seg 5 & seg6 & seg2 & seg3 & seg4 & $\operatorname{seg} 5$ & seg6 & seg2 & seg3 & seg 4 & $\operatorname{seg} 5$ & seg6 \\
\hline segI & 1.00 & 0.82 & 0.82 & 0.82 & 0.82 & 0.94 & 0.82 & 0.82 & 0.61 & 0.73 & 0.94 & 1.00 & 0.82 & 0.59 & 0.59 \\
\hline seg2 & & 0.94 & 0.94 & 1.00 & 0.70 & & 1.00 & 0.73 & 0.39 & 0.59 & & 0.82 & 0.94 & 0.70 & 0.70 \\
\hline seg3 & & & 1.00 & 1.00 & 0.94 & & & 0.94 & 0.59 & 0.82 & & & 1.00 & 0.94 & 1.00 \\
\hline $\operatorname{seg} 4$ & & & & 0.94 & 1.00 & & & & 0.59 & 0.82 & & & & 1.00 & 1.00 \\
\hline $\operatorname{seg} 5$ & & & & & 0.82 & & & & & 0.70 & & & & & 0.94 \\
\hline
\end{tabular}

HFD - Higuchi Fractal Dimension, SPEN - Spectral Entropy, LZC - Lempel-Ziv Complexity, APEN - Approximate Entropy

ment animals by about $1.6^{\circ} \mathrm{C}$ and the heart rate by 14.2 bpm in a population of six anaesthetised pigs. No significant changes were observed in the EEG activity of the pigs although several EEG signal analysis methods were applied. In a population of ten pigs, anaesthetized to the level where the EEG burst suppression pattern occurs, no correlation was found between switching on or off the EMF stimulation and triggering of the bursts.

In this study we wanted to see if RF radiation at high exposure levels could trigger the hypersensitive nonlinear burst suppression control system of anaesthesia EEG. However, in none of our pigs was there any sign of this kind of activation. The results of our study indicate therefore that the $\mathrm{RF}$ radiation does not produce sensory stimulation of somatosensory, auditory or visual system or directly affect the brain so as to produce EEG bursts during suppression. The slow rise in temperature at very high RF intensity suggests that there is an effect also in brain tissue, but this is not appropriate, intensive or abrupt enough to trigger the burst control system.

\section{Competing interests}

The authors declare that they have no competing interests. 

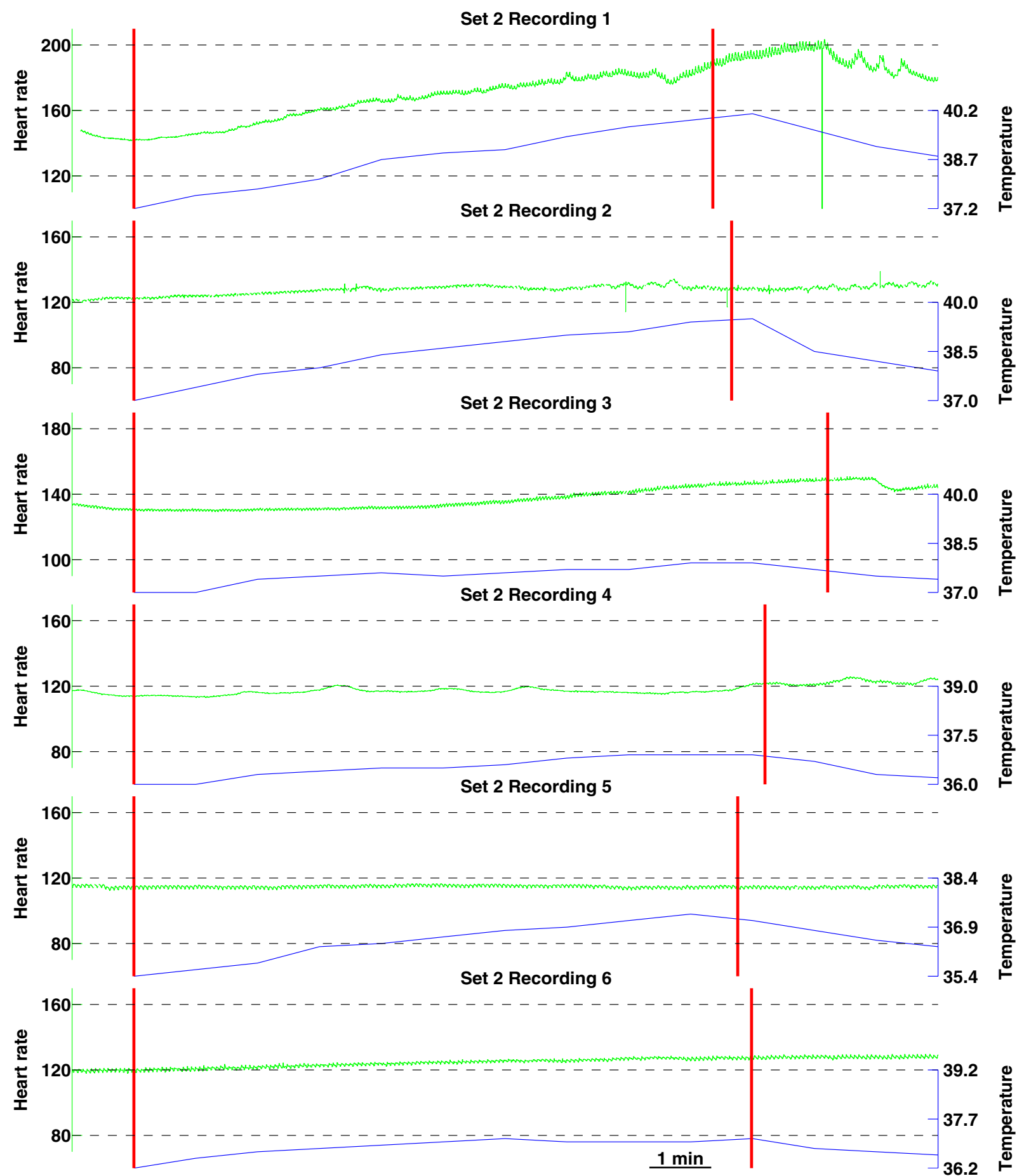

Figure 7

Heart rate (green) and temperature (blue) changes during 10 minute exposure periods in phase II. The time instances at which exposure was switched on or off are marked by vertical lines and the curves are aligned according to the onset of the exposure. The length of the exposure periods varied slightly from recording to recording. Temperature values were written down manually at every minute during (and for several minutes after) the exposure periods. While start- and endpoints of exposure periods are aligned accurately with the heart rate curve, there may be slight (up to half a minute) misalignment between heart rate and temperature. 


\section{Authors' contributions}

MR and KA were responsible for anesthetizing the animals. AM performed the surgical procedures. VJ was responsible for recording the EEG. TT, TToivo and LK were responsible for the dosimetry, design and implementation of the exposure system. RF, TL, AV and VJ performed the data analysis. LK acted as the coordinator of the research team. The manuscript was compiled mostly by TL with the contribution of all the other authors. All authors read and approved the final manuscript.

\section{Acknowledgements}

The assistance of the staff (Laurentiu Barna, Noomi Laakkonen, Leo-Pekka Lyytikäinen, Mikko Myllykoski, Pasi Puumala) of Tampere University of Technology, Tampere University Hospital, Tampere and Faculty of Medicine, University of Tampere is gratefully acknowledged. Special thanks go to the staff of in the test animal laboratory, Faculty of Medicine, University of Tampere. In addition the authors would like to thank Lauri Puranen and Kari Jokela from Radiation and Nuclear Safety Authority (Finland) for their help in dosimetry for exposing anaesthetised pigs and TeliaSonera Finland Oyj for permission to use their frequency channel.

\section{References}

I. Juutilainen J, de Seze R: Biological effects of amplitude-modulated radiofrequency radiation. Scandinavian Journal of Work, Environment \& Health 1998, 24:245-254.

2. Jauchem JR, Ryan KL, Frei MR: Cardiovascular and thermal effects of microwave irradiation at I and/or $10 \mathrm{GHz}$ in anesthetized rats. Bioelectromagnetics 2000, $21: 159-166$.

3. National Radiological Protection Board (presently Health Protection Agency): Health effects from radiofrequency electromagnetic fields. Report of an independent Advisory Group on Nonionising Radiation Documents of the NRPB I4(2). Oxfordshire, UK: Chilton, Didcot; 2003.

4. Lönn S, Ahlbom A, Hall P, Feychting M: Long-term mobile phone use and brain tumor risk. Am J Epidemiol 2005, I6 I (6):526-535.

5. Heikkinen P, Ernst H, Huuskonen H, Komulainen H, Kumlin T, MäkiPaakkanen J, Puranen L, Juutilainen J: No effects of radiofrequency radiation on 3-chloro-4 (dichloromethyl)-5-hydroxy-2(5H)furanone-induced tumorigenesis in female wistar rats. Radiat Res 2006, 166:397-408.

6. Lahkola A, Auvinen J, Raitanen J, Schoemaker MJ, Christensen HC, Feychting M, Johansen C, Klaeboe L, Lönn S, Swerdlow AJ, Tynes T, Salminen T: Mobile phone use and risk of glioma in 5 North European countries. Int J Cancer 2006, I 20: I769-1775.

7. Baranski S, Arber SL, Lin JC: Histological and histochemical effects of microwave irradiation on the central nervous system of rabbits and guinea pigs. Am J Phys Med 1972, 5 I:I82-191.

8. Bawin SM, Gavalas-Medici RJ, Adey WR: Effects of modulated very high frequency fields on specific brain rhythms in cats. Brain Res 1973, 58:365-384.

9. Bawin SM, Gavalas-Medici RJ, Adey WR: Reinforcement of transient brain rhythms by amplitude-modulated VHF fields. In Biological and Clinical Effects of Low Frequency Magnetic and electric Fields Edited by: Llaurado JG, Sances A, Battocletti JH. Springfield, Charles C Thomas; 1974: I72-186.

10. McRee DI, Elder JA, Gage MI, Reiter LW, Rosenstain LS, Shore ML, Galloway WD, Adey WR, Guy AW: Effects of nonionizing radiation on the central nervous system, behaviour and blood: a progress report. Environ Health Perspect 1979, 30:123.

II. Mitchell CL, McRee DI, Peterson NJ, Tilson HA, Shandala MG, Rudnev MI, Varetskii VV, Navakatikyan MI: Results of a United States and Soviet Union joint project on nervous system effects of microwave radiation. Environ Health Perspect 1989, 81:201.

12. Shandala MG, Dumanskii UD, Rudnev MI, Ershova LK, Los IP: Study of nonionizing microwave radiation effects upon the central nervous system and behavior reactions. Environ Health Perspect 1979, 30:115.

13. Thuroczy G, Kubinyi G, Bodo M, Bakos J, Szabo LD: Simultaneous response of brain electrical activity (EEG) and cerebral cir- culation (REG) to microwave exposure in rats. Rev Environ Health 1994, 10:135.

14. International Commission on Non-lonizing Radiation Protection: Guidelines for limiting exposure to time-varying electric, magnetic, and electromagnetic fields (up to $300 \mathrm{GHz}$ ). Health Physics 1998, 74(4):494-522.

15. Servantie B, Servantie AM, Etienne J: Synchronization of cortical neurons by a pulsed microwave field as evidenced by spectral analysis of the electrocorticograms from the white rat. Ann NY Acad Sci 1975, 247:82-86.

16. Takashima S, Onaral B, Schwan HP: Effects of modulated RF energy on the EEG of mammalian brains. Rad Environ Biophys 1979, 16:15-27.

17. Vorobyov VV, Galchenko AA, Kukushkin NI, Akoev IG: Effects of weak microwave fields amplitude modulated at ELF on EEG of symmetric brain areas in rats. Bioelectromagnetics 1997, I8:293-298

18. Hartikainen K: EEG Reactivity during Isofurane-induced Burst Suppression Anaesthesia. In PhD thesis Acta Universitatis Tamperensis; 1996.

19. Hartikainen K, Rorarius M: Cortical responses to auditory stimuli during isoflurane burst suppression anaesthesia. Anaesthesia 1999, 54:210-214.

20. Yli-Hankala $A$, Jäntti $V$, Pyykkö I, Lindgren L: Vibration stimulus induced EEG bursts in isoflurane anaesthesia. Electroencephalogr Clin Neurophysiol 1993, 87:215-220.

21. Jäntti $V$, Yli-Hankala A: Neurophysiology of anaesthesia. Review. In Clinical Neurophysiology in the Beginning of the 2 Ist Century Volume 53. Issue Suppl Edited by: Ambler Z, Nevsimalova S, Kadanka Z, Rossini PM. Elsevier, Clin Neurophysiol; 2000:84-88.

22. Jäntti V, Karvonen E, Puumala P, Sonkajärvi E, Ala-Kokko T: EEG-löydökset iskemian jälkeisessä myokloniassa. Duodecim 2003, I 19:1775-1778. (in Finnish)

23. Jäntti V, Sloan T: EEG and anesthetic effects. Intraoperative Monitoring of Neural Function. Handbook of Clinical Neurophysiology Elsevier 2008, 8(Chapter 4):77-93.

24. Kroeger D, Amzica F: Hypersensitivity of the anesthesiainduced comatose brain. J Neurosci 2007, 27(39): 10597-10607.

25. Hudetz AG, Imas OA: Burst activation of the cerebral cortex by flash stimuli during isoflurane anesthesia in rats. Anesthesiology 2007, 107(6):983-991.

26. Toivonen T, Toivo T, Pitkäaho R, Puranen L, Silfverhuth M, Mennander A, Hannula M, Hyttinen J, Jokela K: Setup and dosimetry for exposing anaesthetised pigs in vivo to $900 \mathrm{MHz}$ GSM mobile phone fields. Bioelectromagnetics 2008, 29:363-370.

27. Inouye T, Shinosaki K, Sakamoto H, Toi S, Ukai S, lyama A, Katsuda Y, Hirano M: Quantification of EEG irregularity by use of the entropy of the power spectrum. Electroencephalogr Clin Neurophysiol 1990, 79:204-210.

28. Pincus SM: Approximate entropy as a measure of system complexity. Proc Nat Acad Sci 1991, 88:2297-2301.

29. Higuchi T: Approach to an irregular time series on the basis of the fractal theory. Physica D 1988, 31:277-283.

30. Lempel A, Ziv J: On the complexity of finite sequences. IEEE Trans Inform Theory 1976, 22:75-8I.

31. Ferenets R, Lipping T, Anier A, Jäntti V, Melto S, Hovilehto S: Comparison of entropy and complexity measures for the assessment of depth of sedation. IEEE Trans Biomed Eng 2007, 53(6): 1067-1077. 